des Digitoxigenins und Gitoxigenins auch in anderen DigitalisArten - Digitalis thapsi, Digitalis lanata - enthalten sind. Inzwischen wurde bekannt, daß Gitoxigenin-mono-digitoxosid auch von D. SATOH u. Mitarb. ${ }^{2}$ ) aus Digitalis purpurea und von J.E. MURPHY ${ }^{3}$ ) aus Digitalis lanata isoliert und Gitorosid bzw. Gitosid benannt worden ist. Die von MuRPHY mitgeteilte Gehaltsbestimmung bestätigt unsere in der 8 . Mitteilung gemachten Angaben, daß der Gehalt an Gitoxigenin-monodigitoxosid in Digitalis lanata höher ist als in Digitalis purpurea.

Wir haben nun gefunden, daß das vierte Hauptgenin der Digitalis-Arten, Digoxigenin, ebenfalls als Mono- und Bisdigitoxosid neben dem bekannten Tri-digitoxosid, Digoxin, in der Natur vorkommt. Durch Chromatographie von Extrakten der fermentierten Blätter von Digitalis lanata an Aluminiumoxyd und mit Formamid imprägniertem Cellulosepulver [Methodik s. 6. und 8. Mitteilung ${ }^{4}{ }^{1}{ }^{1}$ ] gelang es, diese beiden Glykoside rein zu isolieren. Der Gehalt lag in der verwendeten Droge größenordnungsmäßig bei $0,001 \%$ Bis-digitoxosid und $0,0002 \%$ Mono-digitoxosid.

Wir haben Digoxigenin-mono- und -bis-digitoxosid aus Digoxin durch schonende Säurehydrolyse dargestellt und ebenfalls chromatographisch isoliert. Beide Glykoside haben mit den natürlichen Substanzen identische IR-Spektren. Zur Identifizierung der nenen Glykoside auf Papierchromatogrammen und zu ihrer Trennung an Formamidcellulose-Säulen sind die in der Tabelle 1 angegebenen Lösungsmittelgemische geeignet.

Tabelle 1. $R_{f}$-Werte von Digoxigenin-mono- und -bis-digitoxosid im Vergleich mit Digoxin und Digoxigenin. [Lösungsmittel mit Formamid gesättigt, Papier Schleicher \& Schü1l 2043b Mgl, mit Formamid imprägniert $\left.\left.{ }^{5}\right)\right]$

\begin{tabular}{l|c|c|c|c}
\hline Lösungsmittel & Digoxin & \multicolumn{2}{|c|}{ Digoxigenin- } & Digoxi- \\
\cline { 3 - 4 } & $\begin{array}{c}\text { mono-digi- } \\
\text { toxosid }\end{array}$ & $\begin{array}{c}\text { bis-digi- } \\
\text { toxosid }\end{array}$ & genin \\
\hline Chloroform . . . . . & 0,58 & 0,19 & 0,34 & 0,25 \\
CHCl $_{8}$-Isopropyläther 9:1 & 0,60 & 0,23 & 0,41 & 0,25 \\
$\mathrm{CHCl}_{3}$-n-Butyläther 9:1. & 0,37 & 0,11 & 0,21 & 0,14
\end{tabular}

Zur Nomenklatur: Da die Anzahl der Digitalisglykoside - nicht zuletzt auch durch die Aufklärung einer Reihe von Glykosiden des Digitanol-Typs ${ }^{6}$ ) - in den letzten Jahren so groß geworden ist und noch weiter anwachsen wird, erscheint es uns im Interesse der Klarheit und Übersichtlichkeit in der Literatur geraten, für die beiden neuen Glykoside ebenso wie für die 6 in der 8. Mitteilung beschriebenen Digitoxoside, die praktisch $Z$ wischenglieder im Aufbau Genin $\rightarrow$ Tridigitoxosid darstellen, keine neuen, verwirrenden Trivialnamen einzuführen.

Digoxigenin-mono-digitoxosid: $\mathrm{C}_{29} \mathrm{H}_{44} \mathrm{O}_{8}(520,7)$, Ber.: C 66,8, H 8,45, Digitoxose 28,5. Gef.: C 66,63, H 8,51, Digitoxose 28,4 . Schmp. 212 bis $215^{\circ},[\alpha]_{\mathrm{D}}^{20}=+1,4^{\circ} \pm 1^{\circ}$ (Methanol) $\lambda_{\max }=218 \mathrm{~m} \mu \cdot(\log \varepsilon=4,24)$.

Digoxigenin-bis-digitoxosid: $\mathrm{C}_{35} \mathrm{H}_{54} \mathrm{O}_{11}(650,8)$, Ber.: $\mathrm{C} 64,55$ H 8,31, Digitoxose 45,4. Gef. : C 64,63, H 8,71, Digitoxose 43,6. Schmp. 219 bis $222^{\circ},[\alpha]_{D}^{20}=+12,4^{\circ} \pm 1^{\circ}$ (Methanol), $\lambda_{\max }=$ $218 \mathrm{~m} \mu(\log \varepsilon=4,19)$. [Bestimmung der Digitoxose vgl. $\left.{ }^{1}\right)$.]

Forschungslaboratorien der Firma C.F. Boehringer u. Soehne, Mannheim-Waldhof

E. HAACK, F. Katser und H. Spingler

Eingegangen am 26. Oktober 1957

*) 9. Mitteilung über Herzglykoside.

1 KAISER, F., E. HAACK u. H. SPINGLER: Liebigs Ann. Chem. 603, 75 (1957). - ${ }^{2}$ ) Sarom, D., T. WADa u. T. OkuMura: J. Phar mac. Soc. Jap. 76, 1334 (1956). - ${ }^{3}$ ) MurPHY, J. E.: J. Amer. Pharmac. Assoc., Sci. Ed. 46, $170(1957) .-4)$ HAAcxs, E., F. KaISER u. H. SPINGLer: Chem. Ber. 89, 1353 (1956). - 5) KATSER, F. Chem. Ber. 88, 556 (1955). - - $\left.{ }^{6}\right)$ Tschesche, R., u. G. Buschauer: Liebigs Ann. Chem. 603, 59 (1957).

\section{Weitere Kinetin-Analoge}

6-Furylamino-purin (Kinetin) wirkt an pflanzlichen Zellen teilungsauslösend ${ }^{1}$, während an tierischen oder menschlichen Zellen in vitro keine Wirkung erkennbar ist ${ }^{2}$ ). Im $6-\beta$-Indolyläthyl-aminopurin fanden wir die erste Verbindung dieses Typs, die auch bei tierischen und menschlichen Zellen, und zwar sowohl normalen als malignen, in vitro eine Wirkung durch Hemmung des Teilungsablaufes zeigt ${ }^{2}$ ). HAM, EAKIN,
SxINNER und SHIVE ${ }^{3}$ beobachteten eine Hemmwirkung von 6- $\omega$-Phenylalkyl-amino-purinen bei der Regeneration von Hydra. Mit uns von den Autoren überlassenen Präparaten, wofür wir auch hier nochmals danken möchten, konnten wir keine Wirkung dieser Verbindungen auf normale und maligne Zellen in vitro feststellen. Durch Kondensation von Histamin mit 6-Chlor-purin [Methode von BENDRICK, RUSSEL und Fox $\left.\left.{ }^{4}\right)\right]$ haben wir das 6- $\beta$-Imidazolyl-äthylamino-purin dargestellt $^{5}$ ). Diese Verbindung wird von SkINNER ${ }^{6}$ ) ohne weitere Beschreibung erwähnt und als unwirksam im Test der Hemmung der Regeneration von Hydra angegeben. An unseren Objekten in der Gewebekultur zeigt sie eine Besonderheit: sie bewirkt bei acht untersuchten tierischen und menschlichen Carcinomen und Sarkomen in vitro eine starke Zellschädigung und Zellauflösung, während sie gegen normale Zellen ohne Wirkung ist. Die Hemmwirkung dieser Verbindung an Tiertumoren in vivo ist gering, jedoch sei hervorgehoben, daß unter weit über tausend verschiedenen Verbindungen, die wir im Laufe von 18 Jahren an Gewebekulturen untersucht haben und darunter sind praktisch alle je in der Tumortherapie vorgeschlagenen Verbindungen enthalten -, keine bisher eine derartig eindrucksvolle Differenz erkennen ließ.

Vom Purin selbst?) und vom 2,6-Diaminopurin 8) hat BrESELE eine stärkere Wirkung auf Sarkomzellen als auf normale Zellen in vitro mitgeteilt.

Der Yoshida-Tumor der Ratte läßt sich in vitro nur als Dauerkultur züchten, wenn gleichzeitig normale Rattenfibroblasten zugegen sind ${ }^{9}$ ). In dieser Kombination halten wir diesen Tumor jetzt über 3 Jahre in der Gewebekultur. Fügt man das $6-\beta$-Imidazolyl-äthylaminopurin $(100 \% / \mathrm{ml})$ zu einer solchen Mischkultur, so werden nur die Tumorzellen zerstört, und es bieibt eine reine Kultur der normalen Fibroblasten zurück. Diesen Vorgang haben wir im Zeitrafferfilm festgehalten $\left.{ }^{10}\right)$

Es erscheint bemerkenswert, da. biogene Amine wie Tryptamin und Histamin durch die Vereinigung mit der Purinmolekel eine direkte Zellwirkung erlangen. Wir untersuchen daher Purinderivate weiterer biogener Amine.

Fräulein I. Marinius danken wir für ihre fleißige Hilfe bei der Austestung der Verbindungen.

Institut für Experimentelle Kvebsforschung der Universität, Heidelberg

Hans Lettré, Hubert Baliweg und Annelies Schleich Eingegangen am 25. Oktober 1957

1) Milier, C. O., F. Skroog, F. S. Okumura, M. H. v. Saltza 1. F. M. Strong: J. Amer. Chem. Soc. 77, 1392, 2662 (1955); 78 1357 (1956). - - 2) LETTRÉ, H., u. H. ENDo: Naturwiss. 43, 84 (1956). - ") Ham, R. G., R. E. Eakin, C. G. Skinner u. W. Shive: J. Amer. Chem. Soc. 78, 2648 (1956). - 4) BENDRICK, A., P. J. Russel U. J. J. Fox: J. Amer. Chem. Soc. 76, 6073 (1954). 5) BALLWEg, H.: Diplomarbeit, Heidelberg 1957. - 6) SkINNER, C. G. W. ShIVE, R. G. HAM, D. C. FitzGerald U. R. E. EAKIN J. Amer. Chem. Soc. 78, 5097 (1956). - 7) BIEsete, J. J., M. C.

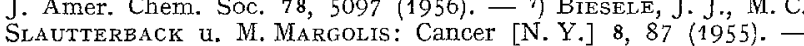
$\left.{ }^{8}\right)$ BIESELE, J. J., R. E. BERGER u. M. ClaAkE: Cancer Res. 12 399 (1952). - 9) LETTRÉ, H., U. A. SCHLEICH: Naturwiss. 41, 505 (1954). - 10) Vorgeführt bei Vorträgen in Bingen am 12. Oktober und in Frankfurt am 15. Oktober 1957.

\section{Über die Henmung proteolytischer Fermente durch Monojodessigsäure}

Im Rahmen unserer Untersuchungen über die Strahlenempfindlichkeit des Gelatine spaltenden Kathepsins (GE) ${ }^{1}$ ) interessierte uns auch die allgemeine Empfindlichkeit dieses Enzyms gegen chemische Agentien. Aus der Literatur war nur bekannt, daß das GE durch $2,5 \times 10^{-4} \mathrm{~m}$ Monojodessigsäure (MES) fast vollständig gehemmt wird ${ }^{2}$ ). Um die Empfindlichkeit der SH-Gruppe des GE mit der von anderen SHEnzymen, etwa von Dehydrogenasen, besser vergleichen zu können, bestimmten wir diejenige Konzentration von MES, die das Ferment zu $50 \%$ hemmt. Aus etwa 30 Bestimmungen bei verschiedenen Konzentrationen wurde jewreils die Regressionsgerade der Fermenthemmung im Probit-logarithmischen Raster berechnet und daraus die $E D_{50}$ mit ihren Fehlergrenzen graphisch ermittelt. Das Ergebnis unserer Untersuchungen zeigt die Tabelle 1.

Das GE ist gegen MES außerordentlich empfindlich. Die MES-Konzentration der 50\% igen Hemmung liegt etwa um 2,5 Größenordnungen tiefer als bei der Triosephosphatdehydrase, die HolzER ${ }^{3}$ ) als das empfindlichste Ferment im Gärungsstoffwechsel angibt ( $50 \%$ Hemmung durch $5 \times 10^{-4} \mathrm{~m}$ MES). Die Hemmung des GE ist unabhängig vom Organ, aus dem das Ferment extrahiert wurde. Glycerin-Rohextrakte 